\title{
Hypersurface Bohm-Dirac models
}

\author{
Detlef Dürr \\ Mathematisches Institut der Universität München, \\ Theresienstraße 39, 80333 München, Germany \\ Sheldon Goldstein \\ Department of Mathematics, Rutgers University, \\ New Brunswick, NJ 08903, USA \\ Karin Münch-Berndl \\ Institut für Angewandte Mathematik, Universität Zürich-Irchel, \\ Winterthurer Strasse 190, 8057 Zürich, Switzerland \\ Nino Zanghì \\ Dipartimento di Fisica, Università di Genova, Sezione INFN Genova, \\ Via Dodecaneso 33, 16146 Genova, Italy
}

(May 28, 1999)

\begin{abstract}
We define a class of Lorentz invariant Bohmian quantum models for $N$ entangled but noninteracting Dirac particles. Lorentz invariance is achieved for these models through the incorporation of an additional dynamical space-time structure provided by a foliation of space-time. These models can be regarded as the extension of Bohm's model for $N$ Dirac particles, corresponding to the foliation into the equal-time hyperplanes for a distinguished Lorentz frame, to more general foliations. As with Bohm's model, there exists for these models an equivariant measure on the leaves of the foliation. This makes possible a simple statistical analysis of position correlations analogous to the equilibrium analysis for (the nonrelativistic) Bohmian mechanics.
\end{abstract}

03.65. Bz

Typeset using REVTEX 


\section{INTRODUCTION}

Among the different approaches to resolving the conceptual problems of quantum theory, Bohm's approach is perhaps the simplest. In a nutshell, it consists in adding the most basic dynamical variables, obeying additional evolution equations, to the description of a quantum system provided by its wave function $\psi$. For nonrelativistic quantum theory the additional variables are the positions of the particles, which evolve according to a "guiding equation" naturally suggested by the Schrödinger evolution. This theory-usually called Bohmian mechanics or the pilot-wave theory - is well understood. It has been analyzed, and its connection with the predictions of orthodox quantum theory explained, in the original papers of Bohm [1] as well as in later works (see, e.g., 20 40 ). One of the main problems remaining for the Bohmian (or any other) approach is to find a satisfactory relativistic quantum theory, a theory that is fully Lorentz invariant while avoiding the profound conceptual difficulties of orthodox quantum theory.

In his original papers, Bohm had an outline for a "Bohmian" field theory, with fields on space-time as the additional variables. A year later he proposed a "Bohmian" model for one Dirac particle [5], which was subsequently extended by Bohm and coworkers to $N$ Dirac particles [6]. For this $N$-particle model the additional variables are, as in Bohmian mechanics, the positions $\mathbf{Q}_{k}, k=1, \ldots, N$, of the particles. However, in contrast with Bohmian mechanics, the guiding equation for this theory

$$
\frac{d \mathbf{Q}_{k}}{d t}=\frac{\psi^{\dagger} \boldsymbol{\alpha}_{k} \psi}{\psi^{\dagger} \psi}
$$

is ultralocal on configuration space: The right hand side of (1) depends only upon the value of $\psi$ at the positions of the particles and not upon spatial derivatives of $\psi$ there. Here $\psi=\psi\left(\mathbf{q}_{1}, \ldots, \mathbf{q}_{N}, t\right)$, taking values in the $N$-particle spin space $\left(\mathbb{C}^{4}\right)^{\otimes N}$, solves the $N$-particle Dirac equation $(\hbar=c=1)$

$$
\begin{array}{r}
i \frac{\partial \psi}{\partial t}=\sum_{k=1}^{N}\left(-i \boldsymbol{\alpha}_{k} \cdot \nabla_{k}-e \boldsymbol{\alpha}_{k} \cdot \mathbf{A}\left(\mathbf{q}_{k}, t\right)\right. \\
\left.+e \Phi\left(\mathbf{q}_{k}, t\right)+\beta_{k} m\right) \psi,
\end{array}
$$

where $\boldsymbol{\alpha}_{k}=\left(\alpha_{k}^{1}, \alpha_{k}^{2}, \alpha_{k}^{3}\right), \alpha_{k}^{i}=I \otimes \cdots \otimes I \otimes \alpha^{i} \otimes I \otimes \cdots \otimes I$, with the $i$-th Dirac $\alpha$ matrix $\alpha^{i}$ at the $k$-th of the $N$ places, and $\beta_{k}$ is defined analogously. $\Phi$ and $\mathbf{A}$ are external electromagnetic potentials. (We may of course consider particle-dependent masses $m_{k}$, charges $e_{k}$, and external potentials $\Phi_{k}$ and $\mathbf{A}_{k}$, but for simplicity we shall not do so.) We shall call this model the Bohm-Dirac model (BD model). Just as with Bohm's proposal for a field theory, the BD model requires for its formulation the specification of a distinguished frame of reference - in terms of which the actual configuration $\left(\mathbf{Q}_{1}, \ldots, \mathbf{Q}_{N}\right)$ and the generic configuration $\left(\mathbf{q}_{1}, \ldots, \mathbf{q}_{N}\right)$ at time $t$ is defined - and in fact the model is not Lorentz invariant if $N>1$ [6].

However, for $N=1$ this model is Lorentz invariant, and may be formulated in a covariant way: Writing $X=X(\tau)$ for the space-time point along a trajectory, with (scalar) parametrization $\tau$, the guiding equation may be written as 


$$
\frac{d X}{d \tau}=j \equiv \bar{\psi} \gamma \psi
$$

with $\psi$ satisfying the Dirac equation

$$
(i \gamma \cdot \partial-e \gamma \cdot A-m) \psi=0
$$

where $\gamma \cdot \partial \equiv \gamma^{\mu} \partial_{\mu}$ and $\gamma \cdot A \equiv \gamma^{\mu} A_{\mu}(x)$. Note that the right hand side of (3), the Dirac current $j=j^{\mu} \equiv \bar{\psi} \gamma^{\mu} \psi$, is the simplest 4-vector that can be constructed from the Dirac spinor $\psi$.

Note also that the parameter $\tau$ has no intrinsic physical significance, so that equation (3) is equivalent to

$$
\frac{d X}{d \tau}=a j
$$

with arbitrary positive scalar field $a=a(x)$. It is not the field of 4 -vectors $j$ (having direction and length) that determines the particle motion, but rather the field of directions defined by $j$. In other words, the law for the particle motion could be formulated in a purely geometrical manner as the condition that the Dirac current $j$ at every point along the trajectory be tangent to the trajectory at that point.

Because the Dirac current is time-like and divergence free,

$$
\partial \cdot j=0
$$

there is a dynamically distinguished probability distribution on the set of particle paths $X(\tau)$ arising from (3). Any distribution on this space of paths can be defined by specifying for the path the crossing probability for some given equal-time surface $\Sigma_{0}$ in some Lorentz frame. (By this crossing probability we mean the distribution of the point through which the path crosses $\Sigma_{0}$, which is the same thing as the probability distribution for the position of the particle in this frame at the given time.) The distinguished distribution is then defined by the crossing probability for $\Sigma_{0}$ given by $\varrho=j^{0}=\psi^{\dagger} \psi$ on $\Sigma_{0}$ (with $\psi$ suitably normalized), which can be written in a covariant manner as $j \cdot n$ where $n$ in the future-oriented unit normal to the surface. For this distribution the crossing probability for any other equal-time surface will also be given by $j \cdot n$, both for the original frame and any other Lorentz frame. We may roughly summarize the situation by saying that for the distinguished probability distribution, quantum equilibrium holds in all Lorentz frames at all times, with the quantum equilibrium distribution given by $\varrho=\psi^{\dagger} \psi$.

More generally, the crossing probability for any space-like hypersurface $\Sigma$ will also be given by $j \cdot n$, with $n=n(x)$ the future-oriented unit normal field to $\Sigma$. Moreover, for any oriented hypersurface $\Sigma$, the crossing measure (a signed measure that need not be normalized), which describes the expected number of signed crossings through area elements of $\Sigma$,

\footnotetext{
${ }^{1}$ The claims in this and the next paragraph follow directly from the application of the divergence theorem (or Stokes' theorem) to an infinitesimally thin tube of paths between $\Sigma_{0}$ (see below) and the relevant hypersurface $\Sigma$.
} 
with negatively oriented crossings counted negatively, is, for the distinguished distribution, also given by $j \cdot n$, with $n=n(x)$ now the positively oriented unit normal field to $\Sigma$.2

The $N$-particle BD model (1) also has a dynamically distinguished probability distribution on paths. As a consequence of (2) $\varrho=\psi^{\dagger} \psi$ satisfies, in the Lorentz frame in which the dynamics is defined, the continuity equation

$$
\frac{\partial \varrho}{\partial t}+\sum_{k=1}^{N} \boldsymbol{\nabla}_{k} \cdot \mathbf{J}_{k}=0
$$

where

$$
\mathbf{J}_{k}=\varrho \mathbf{v}_{k}=\psi^{\dagger} \boldsymbol{\alpha}_{k} \psi
$$

Thus, if the joint probability distribution for the positions of the $N$ particles is given $\varrho=\psi^{\dagger} \psi$ at some time $t=t_{0}$, then, for the corresponding distribution on paths, it will be given by $\varrho=$ $\psi^{\dagger} \psi$ at all times $t$. However, even for this distinguished distribution, quantum equilibrium will not in general hold in other Lorentz frames: The joint distribution of crossings of equal-time surfaces for other frames will in general not be given by $\psi^{\prime \dagger} \psi^{\prime}$ (where $\psi^{\prime}$ is the wave function in the relevant Lorentz frame) [7,8]. Nonetheless, Bohm and coworkers have argued that the observational content of this model is as Lorentz invariant as the covariant formalism of relativistic quantum theory: Since the predictions for results of measurements for this model can be regarded as reflected in the configuration of various devices and registers - and hence can be derived from probabilities for positions given by $\varrho=\psi^{\dagger} \psi$-at a common time in the distinguished frame, these predictions must agree with those of the usual interpretation. Thus no violation of Lorentz invariance can be detected in experiments [6]. (In particular, the identity of the distinguished Lorentz frame cannot be ascertained by means of any possible observation.)

Lorentz invariance is, however, a delicate issue. Indeed, any theory can be made trivially Lorentz invariant (or invariant under any other space-time symmetry), even on the microscopic level, by the incorporation of suitable additional structure [8]. For this reason Bell has stressed that one should consider what he has called "serious Lorentz invariance," a notion, however, that is extremely difficult to make precise in an adequate way [2]. Lacking a general criterion, we may nonetheless begin to get a handle on "serious Lorentz invariance" by analyzing some specific models. If the models involve additional structure, then whether or not we have serious Lorentz invariance will depend, of course, upon the detailed nature of this structure.

In [8] we have considered a model for which the additional structure for a system of $N$ (noninteracting) Dirac particles is provided by a global synchronization among the particles: The trajectories of the particles are such that each one of them at some given space-time point is tangent to a vector field determined, given the wave function, by that point and

\footnotetext{
${ }^{2}$ In this regard it is perhaps worth noting the following: In Minkowski space there is a natural duality between divergence-free vector fields and closed 3-forms. Such a vector field defines a "deterministic" random path, whose "law" is given directly by the vector field, as in (3), and whose statistics are governed by the dual 3-form, in the manner just described.
} 
those points along the trajectories of the other particles with which that point has been "synchronized." This additional synchronization structure is defined implicitly by the equation of motion and the model is not amenable to a statistical analysis in any obvious way. In other words, this model is not statistically transparent (see Section IV of [8]). Nonetheless, even this model provides a counterexample to the widely held belief that a Lorentz invariant Bohmian theory for many particles is impossible (unless only product states are allowed). In this regard, see also the local model of Squires [9].

In this paper we shall analyze a statistically transparent counterexample, the "hypersurface Bohm-Dirac model" (HBD model). The basic idea was proposed in [10] in the context of bosonic quantum field theory: In addition to the wave function and field variables, a distinguished foliation of space-time - a new element of geometrical structure defining simultaneity surfaces - is suggested as an additional dynamical variable of the theory. These surfaces need not be hyperplanes. The defining (Lorentz invariant) equations of the theory should describe the evolution of the wave function, the field variables, and the simultaneity surfaces. For a careful philosophical discussion of how this may be compatible with some appropriate notion of relativity, even if the simultaneity surfaces should turn out to be unobservable, see Maudlin [11].

Here we shall consider such a theory, not for fields but for $N$ (noninteracting) Dirac particles. We shall discuss an as yet incomplete hypersurface Bohm-Dirac model: The law for the evolution of the foliation is not specified, beyond the requirement that it not involve the positions of the particles. We present no hypothesis concerning the origin of the foliation, but have in mind that the foliation should ultimately be governed by a Lorentz invariant law, one that may, for example, involve the $N$-particle wave function. (For definiteness we shall give some very tentative and less than compelling examples of laws for the foliation in Section [V.) However, we show in Subsection IIIA that, regardless of how the foliation is determined, the dynamics of the HBD model preserves the quantum equilibrium distribution on the leaves of the foliation. Thus the model is amenable to the same sort of statistical analysis as for nonrelativistic Bohmian mechanics. This is discussed briefly in Subsection IIIB.

\section{THE HYPERSURFACE BOHM-DIRAC MODEL}

A general foliation $\mathcal{F}$ of codimension one on Minkowski space $M$ can approximately be thought of as a partition of $M$ into 3-dimensional hypersurfaces. These hypersurfaces are the leaves of the foliation. The simplest way to obtain a foliation is by a smooth function $f: M \rightarrow \mathbb{R}$ without critical points, i.e., $d f \neq 0$ everywhere. The level sets $f^{-1}(s)$ are smooth hypersurfaces and form a foliation of $M$. With the one-form $d f_{x}$, which vanishes on the tangent space of the hypersurface through $x \in M$, we may associate by the Lorentz metric the normal vector field $\partial f(x)$. If this is time-like everywhere, and thus the foliation hypersurfaces space-like, we may normalize $\partial f(x)$ to obtain a unit normal vector field $n(x)$ associated with the foliation $\mathcal{F}$.

We shall consider in this paper only space-like foliations, i.e., foliations by space-like hypersurfaces. While obviously different $f$ 's may generate the same foliation $\mathcal{F}$, the futureoriented unit normal vector field $n$ is uniquely determined by $\mathcal{F}$. When does a vector field $v(x)$ determine a foliation $\mathcal{F}$ such that for all $x \in M, v(x)$ is normal to the tangent space 
of the foliation hypersurface through $x$ ? If we denote by $V$ the one-form associated with $v$ by the Lorentz metric, then, by Frobenius' theorem, the necessary and sufficient condition is that $V$ be completely integrable, $V \wedge d V=0$.

Apart from the foliation, the other dynamical variables of the hypersurface Bohm-Dirac model are the usual ones: the wave function $\psi$, here for $N$ Dirac particles, and the $N$-path, the $N$-tuple of (everywhere either time-like or light-like) space-time paths, which describes the trajectories of the $N$ Dirac particles. Covariant laws for these dynamical variables suggest themselves when we write those of the the Bohm-Dirac model, defined by (1) and (2), in a coordinate-free, i.e., covariant manner.

To achieve this we consider first of all the $\psi$-function in the multi-time formalism: For $N$ Dirac particles the wave function $\psi=\psi\left(x_{1}, x_{2}, \ldots, x_{N}\right), x_{k} \in M$, takes values in the $N$-particle spin space $\left(\mathbb{C}^{4}\right)^{\otimes N}$ and satisfies $N$ Dirac equations

$$
\left(i \gamma_{k} \cdot \partial_{k}-e \gamma_{k} \cdot A\left(x_{k}\right)-m\right) \psi=0
$$

$k=1, \ldots, N$. Here $\gamma_{k}=I \otimes \cdots \otimes I \otimes \gamma \otimes I \otimes \cdots \otimes I$, with $\gamma$ at the $k$-th of the $N$ places, and $A$ is an external electromagnetic potential. (Just as with (2), we may of course consider particle-dependent masses $m_{k}$, charges $e_{k}$, and external potentials $A_{k}$.) The system of equations (7) is a covariant version of (2); in this multi-time form the Lorentz invariance of the law for $\psi$ is manifest [6]. The $N$ Dirac particles are coupled by the common wave function $\psi$. If this is entangled, we have nonlocal correlations between the $N$ particles, despite the fact that the particles are noninteracting.

We shall now develop the guiding law for the $N$-path. Note that the numerator of the right hand side of (1) is given by a current $j_{k}$,

$$
j_{k}=\bar{\psi} \gamma_{1}^{0} \ldots \gamma_{k} \ldots \gamma_{N}^{0} \psi
$$

that involves matrix elements of an operator having as factors the 0-component $\gamma^{0}$ of a 4vector for all but the $k$-th particle. Therefore $j_{k}$ can be expressed in a covariant manner by replacing $\gamma_{k}^{0}$ in the above expression with $\gamma_{k} \cdot n$, where $n$ is the future-oriented unit normal to the $t=$ const hyperplanes,

$$
j_{k}=\bar{\psi}\left(\gamma_{1} \cdot n\right) \ldots \gamma_{k} \ldots\left(\gamma_{N} \cdot n\right) \psi
$$

Moreover, the denominator of the right hand side of (11) can be expressed covariantly as $j_{k} \cdot n$. Then the covariant velocity of the $k$-th particle - with respect to the time of a Lorentz frame with $n$ as time axis - is

$$
\frac{d X_{k}}{d t}=\frac{j_{k}}{j_{k} \cdot n}
$$

Since $j_{k} \cdot n=\bar{\psi}\left(\gamma_{1} \cdot n\right) \ldots\left(\gamma_{N} \cdot n\right) \psi$ is independent of $k$, we may reparametrize the paths with a parameter $s$ so related to $t$ that $t^{\prime}(s)=j_{k} \cdot n$ to obtain

\footnotetext{
${ }^{3}$ Note that in the single-time form (2) we can easily add an explicit interaction potential $V\left(\mathbf{q}_{1}, \ldots, \mathbf{q}_{N}, t\right)$ for the $N$ Dirac particles, while in the multi-time form this is impossible.
} 


$$
\frac{d X_{k}}{d s}=j_{k}
$$

More generally, by further reparametrization, we may obtain $d X_{k} / d \tau=a j_{k}$, where $a$ is any positive scalar field. The physical particle dynamics - i.e., the $N$ space-time paths defined by the equations of motion (and initial conditions) - is invariant under reparametrization.

A manifestly "parametrization invariant" formulation of the dynamics - that is, such that a time parameter plays no role - is easily obtained: The space-time paths for the $N$ particles are constrained by the currents $j_{k}$ by requiring that the path for the $k$-th particle at the point $x_{k}$ be tangent to the current $j_{k}$ evaluated at $x_{k}$ and at the the intersection points of the paths of the $N-1$ other particles with the $t=$ const-hyperplane $\Sigma_{t}$ containing $x_{k}$. If we denote by $X_{k}\left(\Sigma_{t}\right)$ the intersection point of the path $X_{k}$ with the hyperplane $\Sigma_{t}$, and by $\dot{X}_{k}\left(\Sigma_{t}\right)$ a tangent of (or the tangent line to) the path $X_{k}$ at $X_{k}\left(\Sigma_{t}\right)$, we may write the law for the $N$-path as

$$
\dot{X}_{k}\left(\Sigma_{t}\right) \| j_{k}\left(X_{1}\left(\Sigma_{t}\right), \ldots, X_{N}\left(\Sigma_{t}\right)\right)
$$

using the symbol || for "is parallel to." In this geometric formulation the Bohm-Dirac dynamics depends upon the Lorentz frame only via its associated foliation into simultaneity hypersurfaces $\Sigma_{t}$, and thus naturally extends to an arbitrary foliation $\mathcal{F}$ of Minkowski spacetime $M$ by curved space-like hypersurfaces:团

Given such a foliation $\mathcal{F}$ and $\Sigma \in \mathcal{F}$, let $X_{k}(\Sigma)$ be the intersection of the path $X_{k}$ with $\Sigma$,円 and let $\dot{X}_{k}(\Sigma)$ be a tangent of (or the tangent line to) the path $X_{k}$ at $X_{k}(\Sigma)$. The law of the $N$-path $X=\left(X_{1}, \ldots, X_{N}\right)$ for the hypersurface Bohm-Dirac model is defined by the currents $j_{k}$ naturally extending (8)

$$
j_{k}=\bar{\psi}\left(\gamma_{1} \cdot n_{1}\right) \ldots \gamma_{k} \ldots\left(\gamma_{N} \cdot n_{N}\right) \psi
$$

where $n_{1} \equiv n\left(x_{1}\right), \ldots, n_{N} \equiv n\left(x_{N}\right)$, with $n$ the future-oriented unit normal vector field associated with $\mathcal{F}$, via the HBD tangency condition (see also Fig. 1)

$$
\dot{X}_{k}(\Sigma) \| j_{k}\left(X_{1}(\Sigma), \ldots, X_{N}(\Sigma)\right) .
$$

[By considering the action of a suitable Lorentz transformation on $\gamma^{0} \gamma \cdot n$ for arbitrary timelike unit vector $n$ (transforming $n$ to $(1,0,0,0)$ ), one sees that $\gamma^{0} \gamma \cdot n$ is a positive operator

\footnotetext{
${ }^{4}$ This is in marked contrast with the parametrized dynamics such as given by equations (9) or (10), which need not extend in anything like the same form to a general foliation since the parametrized paths generated by the dynamics need not, in general, respect the foliation.

${ }^{5}$ Note that the paths $X_{k}$ comprising an $N$-path, since they are nowhere space-like, can intersect $\Sigma$ at most once. This is the main reason why it is important that the foliation $\mathcal{F}$ be space-like. Of course, also from the physical point of view a synchronization along space-like hypersurfaces yields a picture which perhaps makes most sense. We shall assume, without further ado, global existence: that a fragment of an $N$-path locally satisfying the HBD tangency condition, see (13), can be continued in such a manner that each of its paths $X_{k}$ intersects every $\Sigma \in \mathcal{F}$.
} 
in spin space $\mathbb{C}^{4}$. Hence $\left(\gamma_{1}^{0} \gamma_{1} \cdot n_{1}\right) \ldots\left(\gamma_{k}^{0} \gamma_{k} \cdot n\right) \ldots\left(\gamma_{N}^{0} \gamma_{N} \cdot n_{N}\right)$ is also positive, i.e., $j_{k} \cdot n \geq 0$ with "=" only if $\psi=0$. This means that, where it is nonzero, $j_{k}$ is future-oriented and, like the path $X_{k}$, nowhere space-like.]

We may also write down the equations of motion in the parametrized form analogous to (9) or (10). To do so it is convenient to label the hypersurfaces of the foliation using a function $f: M \rightarrow \mathbb{R}$ that generates the foliation as described above, and use this hypersurface labeling as the parameter for the particle trajectories - so that $X_{k}(s)$ is on the hypersurface $f^{-1}(s)$. From the geometrical characterization of the dynamics (13) we know that $d X_{k} / d s$ is parallel to $j_{k}\left(X_{1}(s), \ldots, X_{N}(s)\right)$, and the scale factor required to ensure $f\left(X_{k}(s)\right)=s$ for all $k$ and $s$ is easily seen to be $1 /\left(\partial f \cdot j_{k}\right)$. Therefore

$$
\frac{d X_{k}}{d s}=\frac{j_{k}\left(X_{1}(s), \ldots, X_{N}(s)\right)}{\partial f\left(X_{k}(s)\right) \cdot j_{k}\left(X_{1}(s), \ldots, X_{N}(s)\right)} .
$$

For a flat foliation we may choose a Lorentz frame such that the foliation hyperplanes are the $x^{0}=$ const-planes, i.e. $f(x)=x^{0}$ for all $x$. Then $n=\partial f=(1,0,0,0)$ and (14) reduces to the Bohm-Dirac law (1).

\section{STATISTICAL ANALYSIS OF THE HBD MODEL}

\section{A. Quantum equilibrium}

We shall show now that for the hypersurface Bohm-Dirac model, with foliation $\mathcal{F}$, there is a distinguished probability measure on $N$-paths $X$ satisfying the HBD tangency condition (13), one for which the distribution of hypersurface crossings $X_{1}(\Sigma), \ldots, X_{N}(\Sigma)$ for $\Sigma \in$ $\mathcal{F}$ depends only upon $\psi$ restricted to $\Sigma$ (or, more precisely, to $\Sigma^{N}$ ) for $\psi$ satisfying (7). We shall say that such a distinguished measure, as well as the corresponding hypersurface crossing distribution, is equivariant, defining quantum equilibrium. The physical significance of the hypersurfaces $\Sigma \in \mathcal{F}$ is thus twofold: They serve (via (13)) to define the motion of the particles, and, for a quantum equilibrium $N$-path, it is "on these hypersurfaces" that, manifestly, the "particles are in quantum equilibrium."

The natural candidate for the equivariant crossing probability density $\varrho$ of the HBD model is given by the obvious covariant extension of the equivariant density $\psi^{\dagger} \psi(=$ $\left.\bar{\psi} \gamma_{1}^{0} \ldots \gamma_{N}^{0} \psi\right)$ of the BD model:

$$
\varrho=\bar{\psi}\left(\gamma_{1} \cdot n_{1}\right) \ldots\left(\gamma_{N} \cdot n_{N}\right) \psi
$$

To see that this is in fact equivariant, note the following: In view of (12), (i) $\varrho=j_{k} \cdot n_{k}$ and

$$
j_{k} \cdot n_{k} \text { is independent of } k \text {. }
$$

Furthermore, (ii) the currents $j_{k}$ are divergence free:

$$
\partial_{k} \cdot j_{k}=0
$$

which follows immediately from (12) using the Dirac equation (7) and its adjoint. These two properties of the currents, (16) and (17), are the key ingredients for the proof of the 
equivariance of $\varrho$. For any current satisfying (16) and (17), for the particle dynamics defined by (13), $\varrho=j_{k} \cdot n_{k}$ is an equivariant probability density for crossings of the leaves of the foliation.

The proof of this assertion consists of two steps: First we determine how an arbitrary probability density $R$ on crossings of a foliation hypersurface $\Sigma$ evolves under the dynamics (13), i.e., we formulate the continuity equation of the hypersurface dynamics. In the second step, we show that $R=\varrho$ solves the continuity equation. It then follows that if the probability distribution of the "positions of the $N$ particles" on $\Sigma \in \mathcal{F}$ is given by $\varrho$ restricted to $\Sigma$, then for any other hypersurface $\Sigma^{\prime} \in \mathcal{F}$, the probability distribution of the "positions of the $N$ particles" on $\Sigma^{\prime}$ which emerges by transport according to the dynamics (13) is given by $\varrho$ restricted to $\Sigma^{\prime}$. Thus $\varrho$ is equivariant.

Consider thus two infinitesimally close hypersurfaces $\Sigma$ and $\Sigma^{\prime}$ belonging to the foliation $\mathcal{F}$. The probability distribution of the positions of the $N$ particles on $\Sigma$ is given by a density $R_{\Sigma}: \Sigma^{N} \rightarrow \mathbb{R}$ such that

$$
\begin{array}{r}
\operatorname{Prob}\left(\text { particle } i \text { crosses } \Sigma \text { in } \delta x_{i}, i=1 \ldots N\right) \\
=R_{\Sigma}\left(x_{1}, \ldots, x_{N}\right) \delta x_{1} \cdots \delta x_{N} .
\end{array}
$$

By $\delta x$ we denote simultaneously an infinitesimal region on $\Sigma$ around $x$ and its area (i.e., 3 -volume). Now we compare $R_{\Sigma}$ evaluated at $\left(x_{1}, \ldots, x_{N}\right) \in \Sigma^{N}$ with $R_{\Sigma^{\prime}}$ evaluated at $\left(x_{1}^{\prime}, \ldots, x_{N}^{\prime}\right) \in\left(\Sigma^{\prime}\right)^{N}$, where $x^{\prime} \in \Sigma^{\prime}$ is obtained from $x \in \Sigma$ via displacement from $\Sigma$ to $\Sigma^{\prime}$ in the normal direction, see Fig. 2. Let $\delta x^{\prime}$ be the area of the image of the region $\delta x$ under this correspondence. (Since the projection of the Lorentz metric on $\Sigma^{\prime}$ need not agree with the image, under $x \mapsto x^{\prime}$, of its projection on $\Sigma, \delta x$ and $\delta x^{\prime}$ need not agree.)

Recall from elementary physics that a continuity equation such as (5) is an expression of a local conservation law that, on the infinitesimal level, can be stated as follows: The difference between the probability densities $R_{\Sigma}$ on $\Sigma^{N}$ and $R_{\Sigma^{\prime}}$ on $\left(\Sigma^{\prime}\right)^{N}$ (with $\Sigma^{\prime}$ infinitesimally later than $\Sigma$ ) is accounted for by the flux through the lateral sides - to which the hypersurface normals are tangent - of the configuration-space-time box between $\delta x_{1} \times \ldots \times \delta x_{N} \subset \Sigma^{N}$ and the corresponding set of (primed) points in $\left(\Sigma^{\prime}\right)^{N}$, see Fig. 3;

$$
\begin{gathered}
R_{\Sigma^{\prime}}\left(x_{1}^{\prime}, \ldots, x_{N}^{\prime}\right) \delta x_{1}^{\prime} \cdots \delta x_{N}^{\prime}-R_{\Sigma}\left(x_{1}, \ldots, x_{N}\right) \delta x_{1} \cdots \delta x_{N}= \\
-\sum_{k=1}^{N} \delta x_{1} \ldots \widehat{\delta x_{k}} \cdots \delta x_{N} \int_{\partial\left(\delta x_{k}\right)}\left(R_{\Sigma} v_{k}\right)\left(x_{1}, \ldots, x_{k-1}, y, x_{k+1}, \ldots, x_{N}\right) \cdot\left(u_{k} \delta \tau\right)(y) d S_{k}
\end{gathered}
$$

where the $\widehat{-}$ on $\widehat{\delta x_{k}}$ indicates that this term should be omitted from the product. Here $y$ is the integration variable on $\partial\left(\delta x_{k}\right)$, the (2-dimensional) boundary of $\delta x_{k}$ regarded as a region in $\Sigma, d S_{k}$ is the area element of $\partial\left(\delta x_{k}\right), u_{k}$ is the outward unit normal vector field in $\Sigma$ to $\partial\left(\delta x_{k}\right), \delta \tau(y)$ is the Minkowski distance between $y \in \Sigma$ and the corresponding $y^{\prime} \in \Sigma^{\prime}$ (so that $\left.y^{\prime}=y+\delta \tau(y) n(y)\right)$ and

$$
v_{k}=\frac{j_{k}}{j_{k} \cdot n_{k}}
$$

\footnotetext{
${ }^{6}$ In contrast, the current $j_{k}=\bar{\psi} \gamma_{k} \psi$ we considered in [8] satisfies (17) but not (16).
} 
is the covariant velocity of the $k$-th particle relative to $\Sigma$, see Fig. 2.

Equation (18) is the continuity equation for the HBD model in the "infinitesimally integrated form." It is valid for any hypersurface dynamics defined by (13), regardless of whether the currents $j_{k}$ satisfy (16) and (17). However, as we shall now show, if the currents do satisfy (16) and (17), then $R_{\Sigma}=\left.\varrho\right|_{\Sigma}=\left.\left(j_{k} \cdot n_{k}\right)\right|_{\Sigma}$ satisfies (18).

Sincet

$$
\begin{aligned}
& \varrho\left(x_{1}^{\prime}, \ldots, x_{N}^{\prime}\right) \delta x_{1}^{\prime} \cdots \delta x_{N}^{\prime}-\varrho\left(x_{1}, \ldots, x_{N}\right) \delta x_{1} \cdots \delta x_{N} \\
& =\varrho\left(x_{1}^{\prime}, \ldots, x_{N}^{\prime}\right) \delta x_{1}^{\prime} \cdots \delta x_{N}^{\prime} \\
& -\varrho\left(x_{1}, x_{2}^{\prime} \ldots, x_{N}^{\prime}\right) \delta x_{1} \delta x_{2}^{\prime} \cdots \delta x_{N}^{\prime} \\
& +\varrho\left(x_{1}, x_{2}^{\prime}, \ldots, x_{N}^{\prime}\right) \delta x_{1} \delta x_{2}^{\prime} \cdots \delta x_{N}^{\prime} \\
& -\varrho\left(x_{1}, x_{2}, x_{3}^{\prime}, \ldots, x_{N}^{\prime}\right) \delta x_{1} \delta x_{2} \delta x_{3}^{\prime} \cdots \delta x_{N}^{\prime} \\
& +\cdots+\varrho\left(x_{1}, \ldots, x_{N-1}, x_{N}^{\prime}\right) \delta x_{1} \cdots \delta x_{N-1} \delta x_{N}^{\prime} \\
& -\varrho\left(x_{1}, \ldots, x_{N}\right) \delta x_{1} \cdots \delta x_{N}
\end{aligned}
$$

we obtain in this case for the left hand side of (18) (to leading order)

$$
\begin{aligned}
\sum_{k=1}^{N} & \delta x_{1} \ldots \widehat{\delta x_{k}} \cdots \delta x_{N}\left(j_{k}\left(x_{1}, \ldots, x_{k}^{\prime}, \ldots, x_{N}\right) \cdot n\left(x_{k}^{\prime}\right) \delta x_{k}^{\prime}\right. \\
& \left.-j_{k}\left(x_{1}, \ldots, x_{k}, \ldots, x_{N}\right) \cdot n\left(x_{k}\right) \delta x_{k}\right)
\end{aligned}
$$

while the integrand on the right hand side of (18) becomes $\left(j_{k} \cdot u_{k}\right) \delta \tau d S_{k}$. Thus, subtracting the right hand side of (18) from (21), we obtain (to leading order) the sum over $k$ of the integral of $j_{k}$ over the (outward oriented) boundary of the space-time region above $\delta x_{k}$ between $\Sigma$ and $\Sigma^{\prime}$. But since $j_{k}$ is divergence-free (17), each such term, and hence the sum, vanishes. Thus (18) is satisfied, establishing the equivariance of $\varrho$.

We may also write the continuity equation (18) in a purely local form: Writing

$$
\delta R_{\Sigma}\left(x_{1}, \ldots, x_{N}\right)=R_{\Sigma^{\prime}}^{\prime}\left(x_{1}^{\prime}, \ldots, x_{N}^{\prime}\right)-R_{\Sigma}\left(x_{1}, \ldots, x_{N}\right)
$$

where

$$
\begin{aligned}
& R_{\Sigma^{\prime}}^{\prime}\left(x_{1}^{\prime}, \ldots, x_{N}^{\prime}\right) \delta x_{1} \cdots \delta x_{N} \\
= & R_{\Sigma^{\prime}}\left(x_{1}^{\prime}, \ldots, x_{N}^{\prime}\right) \delta x_{1}^{\prime} \cdots \delta x_{N}^{\prime},
\end{aligned}
$$

and applying Gauss' theorem to the right hand side of (18)

$$
\int_{\partial\left(\delta x_{k}\right)} R_{\Sigma} v_{k} \cdot u_{k} \delta \tau d S_{k}=\operatorname{div}_{k}^{\Sigma}\left(R_{\Sigma} v_{k}^{\Sigma} \delta \tau\left(x_{k}\right)\right) \delta x_{k}
$$

\footnotetext{
${ }^{7}$ Note that this decomposition is possible because $\varrho$ is defined on $M^{N}$ (with $M$ Minkowski space), in contrast with an arbitrary $R=\left(R_{\Sigma}\right)_{\Sigma \in \mathcal{F}}$, defined only for $N$-tuples belonging to $\Sigma^{N}$ for some $\Sigma \in \mathcal{F}$, for which therefore such a decomposition is impossible.
} 
where $\operatorname{div}_{k}^{\Sigma}$ is the divergence with respect to the $k$-th coordinate $x_{k}$ on the Riemannian manifold $\Sigma$ and $v_{k}^{\Sigma}$ is the projection of $v_{k}$ on $\Sigma$, yields

$$
\delta R_{\Sigma}+\sum_{k=1}^{N} \operatorname{div}_{k}^{\Sigma}\left(R_{\Sigma} v_{k}^{\Sigma} \delta \tau_{k}\right)=0
$$

where $\delta \tau_{k} \equiv \delta \tau\left(x_{k}\right)$.

Using this form we may also check the equivariance of $\varrho$. To do so, we first "smoothly" label the hypersurfaces of the foliation $\mathcal{F}$ by a parameter $s \in \mathbb{R}$, increasing in the future direction, which may be called a "time parameter," in terms of which (23) becomes a standard differential equation. The function $f: M \rightarrow \mathbb{R}$ that maps any point $x \in M$ to the label $s$ of the hypersurface $\Sigma_{s}$ to which $x$ belongs generates the foliation in the manner described in Section II. In particular, $\partial f=\|\partial f\| n$, where $n$ is the future-oriented unit normal vector field of $\mathcal{F}$. With $\delta s=\left\|\partial f_{k}\right\| \delta \tau_{k}$, where $\partial f_{k} \equiv \partial f\left(x_{k}\right)$, we get from (19) that

$$
v_{k} \delta \tau_{k}=\frac{j_{k}}{j_{k} \cdot \partial f_{k}} \delta s \equiv \hat{v}_{k} \delta s
$$

with $\hat{v}_{k}=d X_{k} / d s$ the velocity of the $k$-th particle in the parametrized formulation of the dynamics (14).

Consider now a coordinate system adapted to our parametrized foliation $\Sigma_{s}$ : one coordinate is clearly given by $s$, and on one foliation hypersurface we introduce an (arbitrary) coordinate system $p$, which is transported to the other foliation hypersurfaces by the flow along the normal field, yielding the system of coordinates $(s, p)$, allowing us to write $x=(s, p)$ for $x \in M$. Then $x_{k}=\left(s_{k}, p_{k}\right) \in \Sigma_{s} \Leftrightarrow s_{k}=s$, and the relation between $x=(s, p)$ and $x^{\prime}=\left(s^{\prime}, p^{\prime}\right)$ from Fig. 2 becomes $p=p^{\prime}$. Let $\delta p$ be the volume element defined by the $p$-coordinates and let $\delta x=g(p, s) \delta p$. In these adapted coordinates the continuity equation (23) assumes, using (22) and (24), the more standard form

$$
\frac{1}{g_{1} \cdots g_{N}} \frac{\partial\left(g_{1} \cdots g_{N} R_{s}\right)}{\partial s}+\sum_{k=1}^{N} \operatorname{div}_{k}^{\Sigma_{s}}\left(R_{s} \hat{v}_{k}^{\Sigma_{s}}\right)=0
$$

with $R_{s}\left(p_{1}, \ldots, p_{N}\right)=R_{\Sigma_{s}}\left(\left(s, p_{1}\right), \ldots,\left(s, p_{N}\right)\right)$ and $g_{k}=g\left(s, p_{k}\right), k=1, \ldots, N$ (and where $\hat{v}_{k}^{\Sigma_{s}}$ is the projection of $\hat{v}_{k}$ on $\left.\Sigma_{s}\right) \cdot \mathrm{P}^{\mathrm{s}}$

For $R_{s}=\varrho_{s}$, (20) is what lies behind the usual (implication of the) chain rule

$$
\begin{aligned}
& \frac{1}{g_{1} \cdots g_{N}} \frac{\partial\left(g_{1} \cdots g_{N} \varrho_{s}\right)}{\partial s} \\
& =\left.\sum_{k=1}^{N} \frac{1}{g_{k}} \frac{\partial\left(g\left(s_{k}, p_{k}\right) \varrho\left(s_{1}, p_{1}, \ldots, s_{k}, p_{k}\right)\right)}{\partial s_{k}}\right|_{s_{k}=s} .
\end{aligned}
$$

\footnotetext{
${ }^{8}$ This evolution equation depends upon $g$ only through the area-expansion factor arising from the normal flow between hypersurfaces, and thus does not really depend upon the choice of coordinates on the hypersurfaces.
} 
Splitting the 4-divergence into pieces corresponding to variations orthogonal to and variations within $\Sigma_{s}$, we obtain

$$
\operatorname{div} j=\|\partial f\|\left(\frac{1}{g} \frac{\partial}{\partial s}\left(g j^{0}\right)+\operatorname{div}^{\Sigma_{s}}\left(\|\partial f\|^{-1} j^{\Sigma_{s}}\right)\right),
$$

where $j^{0}$ is the normal component of $j, j^{0}=j \cdot n$. Setting $j=j_{k}$ and using $\operatorname{div} j_{k}=0$ (17) we then find with (24) that

$$
\frac{1}{g_{k}} \frac{\partial\left(g_{k} \varrho\right)}{\partial s_{k}}+\operatorname{div}_{k}^{\Sigma_{s}}\left(\varrho \hat{v}_{k}^{\Sigma_{s}}\right)=0
$$

for all $k$. Therefore, in view of (26), summation over $k$ establishes that $R_{s}=\varrho_{s}$ satisfies the HBD continuity equation (25).

\section{B. Comparison with quantum mechanics}

The statistical analysis of the hypersurface Bohm-Dirac model can be based on the assumption that the probability distribution on $N$-paths is given by the equivariant density $\varrho$ (15) on some simultaneity surface $\Sigma$ belonging to the foliation $\mathcal{F}$. Then, by equivariance, the statistical predictions of the HBD model (i.e., the crossing probabilities) agree with the quantum predictions for positions for any hypersurface in $\mathcal{F}$. But what can be said about the statistical predictions concerning a hypersurface which is not part of a member of $\mathcal{F}$ ?

For one particle the situation is very simple: From the geometrical formulation of the HBD model (Section III) it follows immediately that the HBD model for one particle is foliation-independent, and in fact is the usual one-particle Bohm-Dirac theory given by eqs. (3) and (4), with current $j=\bar{\psi} \gamma \psi$. Thus in this case the statistical predictions of the model agree with the quantum predictions for position along any hypersurface.

The situation is analogous for $N$ independent particles: If the wave function $\psi$ is a product wave function, $\psi=\psi_{1}\left(x_{1}\right) \cdots \psi_{N}\left(x_{N}\right)$, then it follows from the multi-time Dirac equation (17) that $\psi_{k}$ satisfies the usual one-particle Dirac equation. Furthermore, the path of the $k$-th particle is tangent to the one-particle current $\bar{\psi}_{k} \gamma \psi_{k}$ and thus independent of the paths of the other particles. Moreover $\varrho$ is the product of the corresponding 1-particle distributions. Therefore, a product wave function indeed generates a foliation-independent motion, the motion of $N$ independent Bohm-Dirac particles, and we thus have agreement with all the quantum position distributions in this case.

In the general case the situation is more subtle: If the $N$-particle wave function is entangled, it will not in general be the case that the distribution of crossings of hypersurfaces not belonging to the foliation agree with the corresponding quantum position distributions [7],8 (which, in fact, may be incompatible with the crossing statistics for any trajectory model whatsoever). However, this disagreement does not entail violations of the quantum predictions, as has been discussed for the case of the multi-time translation invariant Bohmian theory in [8]. In fact, insofar as results of measurement are concerned, the predictions of our

model are the same as those of orthodox quantum theory, for positions or any other quantum 
observables, regardless of whether or not these observables refer to a common hypersurface belonging to $\mathcal{F}$.

This is because the outcomes of all quantum measurements can ultimately be reduced to the orientations of instrument pointers, counter readings, or the ink distribution of computer printouts, if necessary brought forward in time to a common hypersurface in $\mathcal{F}$, or even to a single common location, for which agreement is assured. Nonetheless, this situation may seem paradoxical if we forget the non-passive character of measurement in quantum mechanics. The point is that for Bohmian quantum theory, measurement can effect even distant systems, so that the resulting positions - and hence their subsequently measured values - are different from what they would have been had no measurement occurred.

\section{PERSPECTIVE}

We have presented a hypersurface Bohm-Dirac model for $N$ entangled but noninteracting Dirac particles. This model is a covariant extension of the Bohm-Dirac model, which involves a foliation by equal-time (flat) hypersurfaces, to arbitrarily shaped (smooth) hypersurfaces. How natural is this model?

When looking for a relativistic extension of nonrelativistic Bohmian mechanics one inevitably encounters two central, very different problems: that such an extension must involve a mechanism for nonlocal interactions between the particles, and that quantum equilibrium cannot hold in all Lorentz frames. For both of these problems the additional space-time structure provided by a foliation yields the most obvious solution: The motion of each particle at a point $x \in M$ depends upon the paths of the other particles via the points at which they intersect the leaf of the foliation containing $x$, and we have an equivariant density on the leaves of the foliation.

And the simplest way to achieve this, in a covariant manner, for a Dirac wave function $\psi$, is via the current (12): Form the natural tensor $\bar{\psi} \gamma_{1} \ldots \gamma_{N} \psi$, evaluated at $x$ and the other intersection points, and contract in the slots corresponding to the other particles with the $N-1$ unit normals to the hypersurface at the corresponding points, to obtain the divergencefree 4 -vector $j_{k}$, the tangent to the trajectory at $x$. Thus, the dynamics of the HBD model is the simplest Lorentz invariant dynamics compatible with the structure at hand, namely, the Dirac wave function and the foliation. Furthermore, the simultaneous normal component $\varrho=j_{k} \cdot n_{k}$ is an equivariant density on the leaves of the foliation.

It should be stressed, however, that the Lorentz invariance of the HBD model is - in Bell's sense - "serious" only if the foliation is regarded as an additional objective dynamical -in contrast to absolute - structure in the theory (and in the world, if the theory is to describe the world). It is this structure that is the innovation of what has been proposed here and

\footnotetext{
${ }^{9}$ This conclusion requires the rather dubious assumption that the relevant measurements can be understood in terms of noninteracting Dirac particles. However, in order to talk coherently about the quantum predictions for a model, it must be possible to understand measurement processes in terms of that model. The remarks we are making here would also be appropriate for the more realistic models for which this would be true.
} 
in [10], not the model per se, which is indeed a rather straightforward covariant extension of the BD model.

However, in this paper we shall not try to find a "serious" law for the foliation $\mathcal{F}$ or, what amount to the same thing, its normal vector field $n$. As a toy example, however, the foliation law could be given by an autonomous equation for $n$, such as $\partial_{\nu} n^{\mu}=0$. Another class of toy examples involves a vector field $n$ constructed from the wave function $\psi\left(x_{1}, \ldots, x_{N}\right)$ : Consider the space-time vector fields $v_{k l}^{\mu}(x)=\left(\bar{\psi} \gamma_{k}^{\mu} \psi\right)\left(\widehat{x_{1}}, \ldots, \widehat{x_{l-1}}, x, \widehat{x_{l+1}}, \ldots, \widehat{x_{N}}\right)$, where $\left(\widehat{x_{1}}, \ldots, \widehat{x_{N}}\right)$ is a point fixed in a Lorentz invariant way, for example as a maximum of $\bar{\psi} \psi$. (Simply considering $v_{k}^{\mu}(x)=\left(\bar{\psi} \gamma_{k}^{\mu} \psi\right)(x, \ldots, x)$ is not a good idea, since this will be zero for antisymmetric (fermion) wave functions.) Now one may set $n$ equal to the integrable of some $v_{k l}$.

A further possibility, which may be more serious, is to have, in addition to the particle degrees of freedom, an independent quantum field $\phi_{\mu}$ that determines the foliation. Assume that for any quantum state $\Phi$ of the field, $\left(\Phi, \phi_{\mu} \Phi\right)$ is time-like and completely integrable. Then for any state $\Psi$ of the particle-field system, set $n_{\mu}=\left(\Psi, \phi_{\mu} \Psi\right)$. Suppose that the particle and the field degrees of freedom are both dynamically and statistically independent, i.e., that there is neither quantum interaction nor entanglement between these degrees of freedom, so that in particular the full wave function $\Psi=\psi \otimes \Phi$. Then we may define the foliation by the normal field $n_{\mu}$. The $\phi$-field can be regarded as very roughly analogous to a Higgs field, producing a kind of spontaneous symmetry breaking, where by choice of $\Phi$ a particular foliation is determined, and relativistic invariance thereby broken.

\section{ACKNOWLEDGMENTS}

We thank Folker Schamel for helpful discussions. This work was supported in part by the DFG, by NSF Grant No. DMS-9504556, by Swiss NF grant 20-55648.98, and by the INFN.

\footnotetext{
${ }^{10}$ For an arbitrary vector field $v^{\mu}(x)$, the Fourier transformed $\hat{v}^{\mu}(k)$ may be split into $\hat{v}_{\|}^{\mu}(k)=$ $\hat{v}^{\nu}(k) k_{\nu} k^{\mu} /\left(k_{\lambda} k^{\lambda}\right)$ and $\hat{v}_{\perp}^{\mu}(k)=\hat{v}^{\mu}(k)-\hat{v}_{\|}^{\mu}(k)$. The inverse Fourier transformed $v_{\|}^{\mu}(x)$ satisfies the integrability condition $\partial_{\mu} v_{\| \nu}-\partial_{\nu} v_{\| \mu}=0$.
} 


\section{REFERENCES}

[1] D. Bohm, Phys. Rev. 85, 166 (1952); 85, 180 (1952).

[2] J.S. Bell, Speakable and Unspeakable in Quantum Mechanics (Cambridge University Press, Cambridge, 1987).

[3] D. Dürr, S. Goldstein, and N. Zanghì, J. Stat. Phys. 67, 843 (1992).

[4] J.T. Cushing, A. Fine, and S. Goldstein, Bohmian Mechanics and Quantum Theory: An Appraisal (Kluwer Academic, Dordrecht, 1996).

[5] D. Bohm, Prog. Theor. Phys. 9, 273 (1953).

[6] D. Bohm and B.J. Hiley, The Undivided Universe: An Ontological Interpretation of Quantum Theory (Routledge, London, 1993).

[7] T.M. Samols, J. Stat. Phys. 80, 793 (1995).

[8] K. Berndl, D. Dürr, S. Goldstein, and N. Zanghì, Phys. Rev. A 53, 2062 (1996).

[9] E. Squires, Phys. Lett. A 178, 22 (1993).

[10] D. Dürr, S. Goldstein, and N. Zanghì, in Stochastic Processes, Physics and Geometry, edited by S. Albeverio, G. Casati, U. Cattaneo, D. Merlini, and R. Moresi (World Scientific, Singapore, 1990), p. 374.

[11] T. Maudlin, in 44, p. 285.

[12] M. Daumer, D. Dürr, S. Goldstein, and N. Zanghì (unpublished). 


\section{FIGURES}

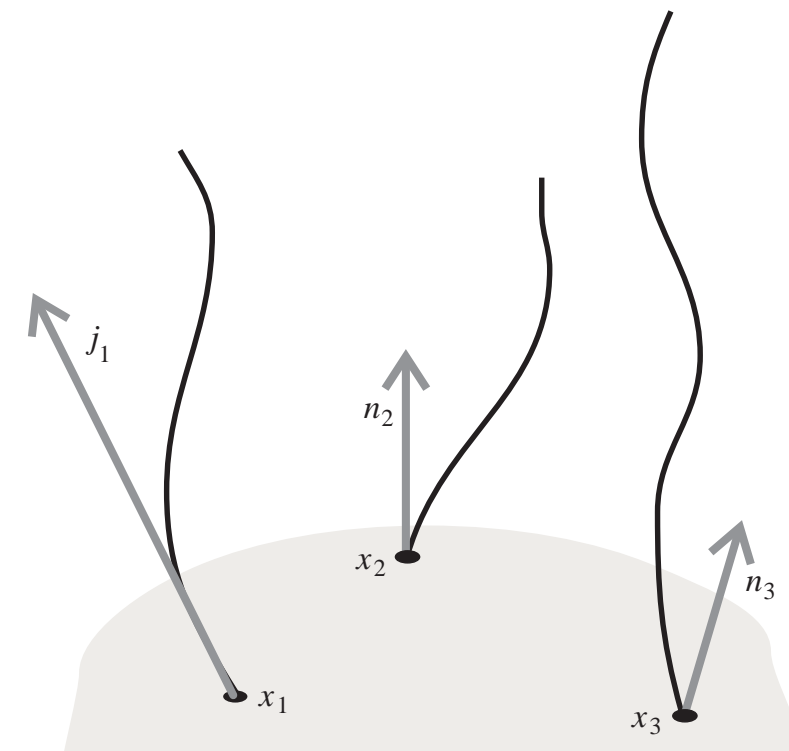

$\Sigma$
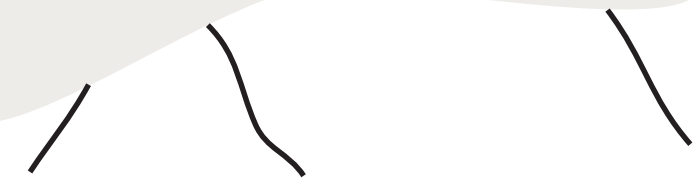

FIG. 1. Geometrical formulation of the dynamics for a system of three particles: For each particle the path of that particle, say particle 1 at $x_{1}$, must be tangent to the 4 -vector $j_{1}$ which is determined by: 1) the intersections $x_{2}$ and $x_{3}$ of the trajectories of the other two particles with the hypersurface $\Sigma$ containing $\left.x_{1}, 2\right)$ the future-oriented unit normals $n_{2}$ and $n_{3}$ at these points, and 3) the wave function of the system evaluated at $x_{1}, x_{2}$ and $x_{3}$ : $j_{1}=\bar{\psi}\left(x_{1}, x_{2}, x_{3}\right) \gamma_{1}\left(\gamma_{2} \cdot n_{2}\right)\left(\gamma_{3} \cdot n_{3}\right) \psi\left(x_{1}, x_{2}, x_{3}\right)$. 


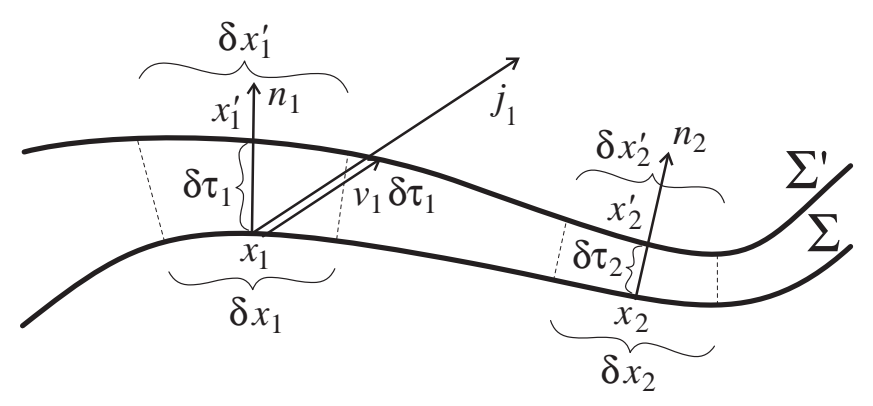

FIG. 2. Motion of two particles in one space dimension from hypersurface $\Sigma$ to $\Sigma^{\prime}$ : space-time view. We have indicated the positions of the primed points $x_{k}^{\prime}$ obtained from $x_{k}$ via displacement from $\Sigma$ to $\Sigma^{\prime}$ in the normal direction, and the images $\delta x_{k}^{\prime}$ of the regions $\delta x_{k}$ under this correspondence. The point on $\Sigma^{\prime}$ to which particle $k$ moves when starting at $x_{k} \in \Sigma$ is given (to leading order) by $x_{k}+v_{k} \delta \tau_{k}$ with $v_{k}=j_{k} /\left(j_{k} \cdot n_{k}\right)$, where $\delta \tau_{k}$ is the Minkowski distance between $x_{k}$ and $x_{k}^{\prime}$.

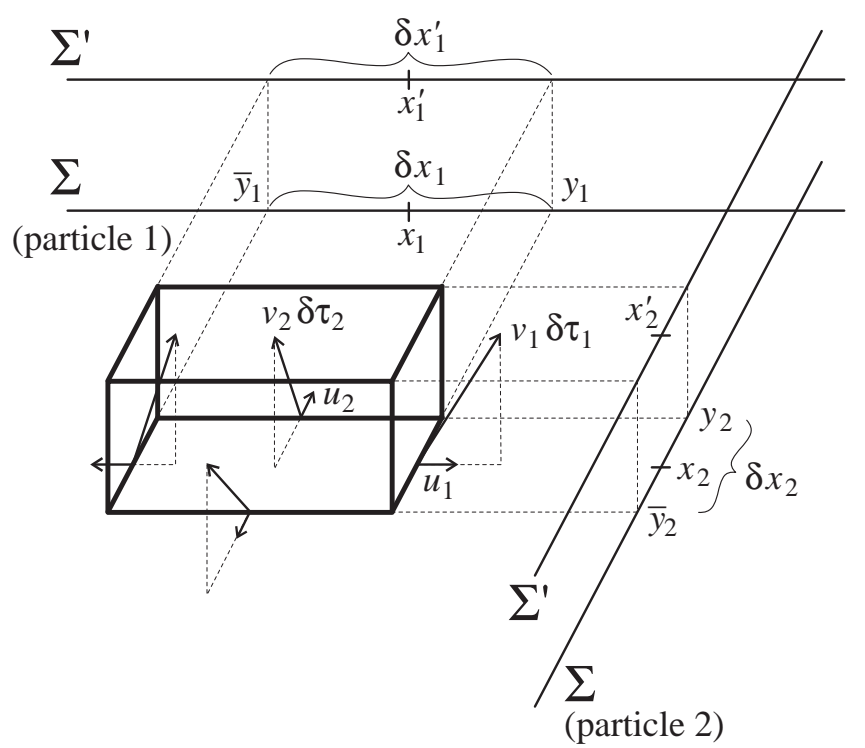

FIG. 3. Conservation of probability for a system of two particles in one space dimension: configuration-space-time view with, for simplicity, the hypersurfaces drawn straightened out. (Note that the figure fails to convey the fact-displayed in Fig. 2 that the areas $\delta x_{k}$ and $\delta x_{k}^{\prime}$ may differ, and that also $\delta \tau\left(y_{k}\right)$ may differ from $\delta \tau\left(\bar{y}_{k}\right)$, where $y_{k}$ and $\bar{y}_{k}$ are the boundary points of $\delta x_{k}$.) The change of the probability of particle 1 being in $\delta x_{1}$ and particle 2 being in $\delta x_{2}$ from hypersurface $\Sigma$ to $\Sigma^{\prime}$ is accounted for by the single particle fluxes through the lateral sides of the configuration space time box between $\delta x_{1} \times \delta x_{2} \subset \Sigma^{2}$ and the corresponding set of primed points on $\left(\Sigma^{\prime}\right)^{2}$, i.e., $R_{\Sigma^{\prime}}\left(x_{1}^{\prime}, x_{2}^{\prime}\right) \delta x_{1}^{\prime} \delta x_{2}^{\prime}-R_{\Sigma}\left(x_{1}, x_{2}\right) \delta x_{1} \delta x_{2}=$ $-\left(\left(R_{\Sigma} v_{1}\right)\left(\bar{y}_{1}, x_{2}\right) \cdot\left(u_{1} \delta \tau\right)\left(\bar{y}_{1}\right)+\left(R_{\Sigma} v_{1}\right)\left(y_{1}, x_{2}\right) \cdot\left(u_{1} \delta \tau\right)\left(y_{1}\right)\right) \delta x_{2}$ $-\left(\left(R_{\Sigma} v_{2}\right)\left(x_{1}, \bar{y}_{2}\right) \cdot\left(u_{2} \delta \tau\right)\left(\bar{y}_{2}\right)+\left(R_{\Sigma} v_{2}\right)\left(x_{1}, y_{2}\right) \cdot\left(u_{2} \delta \tau\right)\left(y_{2}\right)\right) \delta x_{1}$. Eq. (18) is the natural extension of this formula to $N$ particles in Minkowski space. 